\title{
Hydrodynamic Pressure Evaluation of Reservoir Subjected to Ground Excitation Based on SBFEM
}

\author{
Shangming Li \\ Institute of Structural Mechanics, China Academy of Engineering Physics \\ Mianyang City, Sichuan Province
}

China

\section{Introduction}

Dynamic responses of dam-reservoir systems subjected to ground motions are often a major concern in the design. To ensure that dams are adequately designed for, the hydrodynamic pressure distribution along the dam-reservoir interface must be determined for assessment of safety.

Due to the fact that analytical methods are not readily available for dam-reservoir systems with arbitrary geometry shape, numerical methods are often used to analyze responses of dam-reservoir systems. In numerical methods, dams are often discretized into solid finite elements through Finite Element Method (FEM), while the reservoir is either directly modeled by Boundary Element Method (BEM) or is divided into two parts: a near field with arbitrary geometry shape and a far field with a uniform cross section. The near field is discretized into acoustic fluid finite elements by using FEM or boundary elements by BEM, while the far field is modeled by BEM or a Transmitting Boundary Condition (TBC). Based on these numerical methods, several coupling procedures were developed.

A FEM-BEM coupling procedure was used to implement the linear and non-linear analysis of dam-reservoir interaction problems (Tsai \& Lee, 1987; Czygan \& Von Estorff, 2002), respectively, in which the dam was modeled by FEM, while the reservoir was modeled by BEM. A BEM-TBC coupling method was adopted to solve dam-water-foundation interaction problems and dam-reservoir-sediment-foundation interaction problems (Dominguez \& Maeso, 1993; Dominguez et al., 1997). The dam and the near field of the reservoir were discretized by using BEM, while the far field of the reservoir was represented by a TBC. As a traditional numerical method, BEM has been popular in simulating unbounded medium, but it needs a fundamental solution and includes a singular integral, which affect its application. In order to avoid deriving a fundamental solution required in BEM, the TBC attracted some researchers' interests. A Sommerfeld-type TBC was used to represent the far field (Kucukarslan et al., 2005), while a Sharan-type TBC was proposed for infinite fluid (Sharan, 1987). The Sommerfeld-type and Sharan-type TBCs are readily implemented in FEM due to their conciseness, but a sufficiently large near field is required to model accurately the damping effect of semi-infinite reservoir. Except for the aforementioned TBCs, an exact TBC (Tsai \& Lee, 1991), a novel TBC (Maity \& 
Bhattacharyya, 1999) and a non-reflecting TBC (Gogoi \& Maity, 2006) were proposed, respectively. These complicated TBCs gave better results even when a small near field was chosen, but their implementations in a finite element code became complex and tedious.

In this chapter, the scaled boundary finite element method (SBFEM) was chosen to model the far field. The SBFEM does not require fundamental solutions and is able to model accurately the damping effect of semi-infinite reservoir and incorporate with FEM readily, but the SBFEM requires the geometry of far field is layered (or tapered). Although BEM and some of TBCs can handle far fields with arbitrary geometry, far fields in most dam-reservoir systems are always chosen to be layered with a uniform cross section, which ensures the SBFEM can be used in dam-reservoir interaction problems.

Based on a mechanically-based derivation, the SBFEM was proposed for infinite medium (Wolf \& Song, 1996a; Song \& Wolf, 1996) which was governed by a three-dimensional scalar wave equation and a three-dimensional vector wave equation, respectively. A dynamic stiffness matrix and a dynamic mass matrix were introduced to represent infinite medium in the frequency domain and the time domain, respectively. The dynamic stiffness matrix satisfies a non-linear ordinary differential equation of first order, while the dynamic mass matrix is governed by an integral convolution equation. The SBFEM reduces spatial dimensions by one. Only boundaries need discretization and its solutions in the radial direction are analytical. Therefore, it can handle well bounded domain problems with cracks and stress singularities and unbounded domain problems including infinite soil or unbounded acoustic fluid medium. In analyzing crack and stress singularities problems, the SBFEM placed the scaling center on the crack tip and only discretized the boundary of bounded domain using supper elements except the straight traction free crack faces, which permitted a rigorous representation of the stress singularities around the crack tip (Song, 2004; Song \& Wolf, 2002; Yang \& Deeks, 2007). The response of unbounded domain problems was obtained by using the SBFEM alone or coupling FEM and the SBFEM. A FEM-SBFEM coupling procedure was used to analyze unbounded soil-structure interaction problems in the time domain (Ekevid \& Wiberg, 2002; Bazyar \& Song, 2008). For unbounded acoustic fluid medium problems, a FEMSBFEM coupling procedure combined with acoustic approximations was proposed to evaluate the responses of submerged structures subjected to underwater shock waves in the time domain (Fan et al., 2005; Li \& Fan, 2007). Results showed that the SBFEM was able to model accurately the damping behavior of the unbounded soil and infinite acoustic fluid medium, but it was computationally expensive because the evaluations of the dynamic mass matrix and dynamic responses need solving integral convolution equations. In the frequency domain, dynamic condensation and substructure deletion methods were used to evaluate the dynamic stiffness matrix, which avoid evaluating integral convolution equations, but evaluation errors increased with frequency increasing so that results at high frequencies were not acceptable (Wolf \& Song, 1996b). To evaluate accurately high frequencies behaviors of the dynamic stiffness matrix, a Pade series was presented to analyze out-of-plane motion of circular cavity embedded in full-plane through using the SBFEM alone (Song \& Bazyar, 2007). Good results were obtained at high frequencies, but results at low frequencies were inferior even if a high order Pade series was used. The high order Pade series was not only complex, and also increased computational cost. A simplified SBFEM formulation was presented through discovering a zero matrix and a FEM-SBFEM coupling procedure was used to analyze dam-reservoir interaction problems subjected to ground motions (Fan \& Li, 2008). The simplified SBFEM 
was well suitable for all frequencies and no additional computational costs were increased for low frequency analysis in comparison with for high frequency analysis. Its advantages were exhibited by analyzing the harmonic responses of dam-reservoir systems in the frequency domain. However in the time domain, its advantages are not as obvious as those in the frequency domain because integral convolutions still need evaluating. Although a Riccati equation and Lyapunov equations were presented to solve the integral convolutions (Wolf \& Song, 1996b), solving them needed great computational costs, especially for large-scale systems, which limited the SBFEM applications in the time domain. To simplify the integral convolutions and save computational costs, some recursive formulations were proposed (Paronesso \& Wolf, 1998; Yan et al., 2004), based on a diagonalization procedure and the linear system theory (Paronesso \& Wolf, 1995). The integral convolution was transformed into an equivalent system of linear equations, named state-variable description which was represented by finite-difference equations. However, the coefficient matrix quaternion of finite-difference equations was calculated by using Hankel matrix realization algorithms, which complicated the analysis. Furthermore, the diagonalization procedure increased the order of the dynamic mass matrix, and some global lumped parameters, such as springs, dashpots and masses, used in the diagonalization procedure must be introduced at additional internal nodes corresponding to inner variables in the state-variable description, besides the nodes on the structure-medium interface. The number of global lumped parameters would become very large for large-scale systems. This weakened the feasibility of the diagonalization procedure. A new diagonalization procedure of the SBFEM for semi-infinite reservoir was proposed (Li, 2009), whose calculation efficiency was proven to be high, although it still included convolution integrals. With the improvement of the SBFEM evaluation efficiency in the time domain analysis, the SBFEM will show gradually its advantages and potential to solve problems including unbounded soil or unbounded acoustic fluid medium, such as the dam-reservoir interaction problems.

\section{Problem statement}

Consider dam-reservoir interaction problems subjected to horizontal ground accelerations. The dam-reservoir system and its Cartesian coordinate system were shown in Fig.1. The

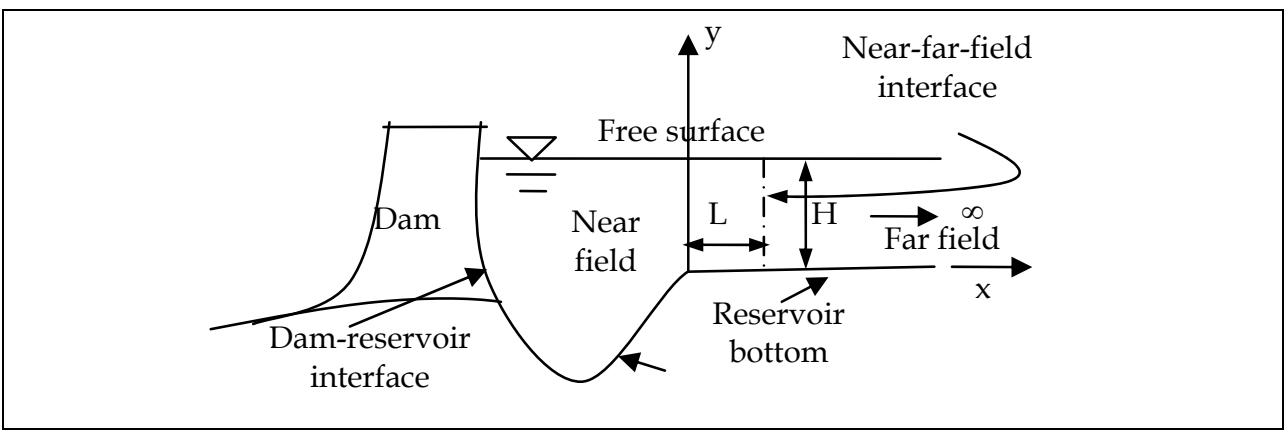

Fig. 1. Dam-reservoir system 
dam was subjected to a horizontal ground acceleration $a_{x}$ and the semi-infinite reservoir was filled with an inviscid isentropic fluid. To evaluate the response of the dam-reservoir system under a horizontal ground acceleration $a_{x}$ excitation, the semi-infinite reservoir was divided into two parts: a near field and a far field. The near field was located between the dam-reservoir interface and the radiation boundary (the near-far-field interface at $x=L$ ), while the far field was from $x=L$ to $\infty$. Note that the geometry of the reservoir was chosen to be arbitrary for $x<0$ and flat for $x \geq 0$.

For an inviscid isentropic fluid (acoustic fluid) with the fluid particles undergoing only small displacements and not including body force effects, the governing equations is expressed as

$$
\nabla^{2} \phi=\frac{1}{c^{2}} \ddot{\phi}
$$

where $\phi$ denotes velocity potential and $c$ denotes the sound speed in fluid. Reservoir pressure $p$, the velocity vector $\mathbf{v}$ and the velocity potential $\phi$ have a relationship as follows:

$$
\begin{aligned}
& \mathbf{v}=\nabla \phi \\
& p=-\rho \dot{\phi}
\end{aligned}
$$

where $\rho$ denotes fluid density. Boundary conditions of the near field for Eq.(1) are following. Along the dam-reservoir interface, one has

$$
\mathbf{v} \bullet \mathbf{n}=\frac{\partial \phi}{\partial n}=v_{n}
$$

where the unit vector $\mathbf{n}$ is perpendicular to the dam-reservoir interface and points outward of fluid; $v_{n}$ is the normal velocity of the dam-reservoir interface. The boundary condition along the reservoir bottom is

$$
\frac{\partial \phi}{\partial n}+q \dot{\phi}=v_{n}
$$

where $q$ is defined as

$$
q=\frac{1}{c}\left(\frac{1-\alpha_{r}}{1+\alpha_{r}}\right)
$$

in which $\alpha_{r}$ denotes a reflection coefficient of pressure striking the bottom of the reservoir. By ignoring effects of surface waves of fluid, the boundary condition of the free surface is taken as

$$
\phi=0
$$

The boundary condition on the radiation boundary (near-far-filed interface) should include effects of the radiation damping of infinite reservoir and those of energy dissipation in the reservoir due to the absorptive reservoir bottom. To model these effects accurately, the SBFEM was adopted in this chapter. 


\section{SBFEM formulation}

Fig.2 showed the SBFEM discretization model of the far field shown in Fig.1, which was a layered semi-infinite fluid medium whose scaling center was located at minus infinity. The whole semi-infinite layered far field was divided into some layered sub-fields. Each layered sub-field was represented by one element on the near-far-field interface, so the whole far field was discretized into some elements on the near-far-field interface. Based on the discretization, a dynamic stiffness or mass matrix was introduced to describe the characteristics of the far field in the SBFEM. The interaction between the near field and the far field was expressed as the following SBFEM formulation.

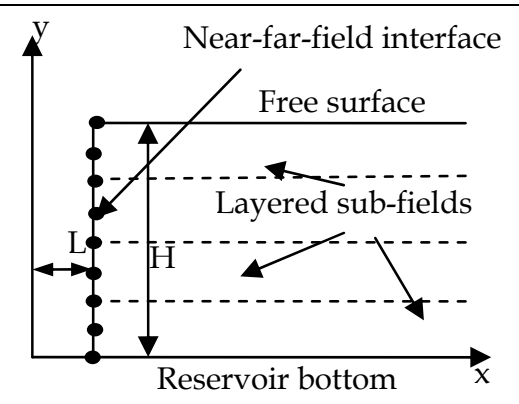

Fig. 2. SBFEM discretization model of layered far field

\subsection{SBFEM formulation in the frequency domain}

On the discretized near-far-field interface, the SBFEM formulation in the frequency domain (Fan \& Li, 2008; Li et al., 2008) for the far field filled with unbounded acoustic fluid medium is written as

$$
\mathbf{V}_{n}(\omega)=\mathbf{S}^{\infty}(\omega) \boldsymbol{\Phi}(\omega)
$$

where $\boldsymbol{\Phi}(\omega)$ denotes the column vector composed of nodal velocity potentials $\phi ; \mathbf{S}^{\infty}(\omega)$ is the dynamic stiffness matrix of the far field and $\mathbf{V}_{n}(\omega)$ satisfies

$$
\mathbf{V}_{n}(\omega)=\sum_{e} \int_{\Gamma_{w}^{e}} \mathbf{N}_{f}^{T} v_{n}(\omega) d \Gamma_{w}^{e}
$$

in which $v_{n}$ is the normal velocity; $\Gamma_{w}$ denotes the near-far-field interface; $\mathbf{N}_{f}$ is the shape function for a typical discretized acoustic fluid finite element; and $\Sigma_{e}$ denotes an assemblage of all fluid elements on the near-far-field interface. The dynamic stiffness matrix $\mathbf{S}^{\infty}(\omega)$ (Li, et al., 2008) satisfies

$$
\left(\mathbf{S}^{\infty}(\omega)+\mathbf{E}^{1}\right) \mathbf{E}^{0-1}\left(\mathbf{S}^{\infty}(\omega)+\mathbf{E}^{1 T}\right)-\mathbf{E}^{2}-i \omega \mathbf{C}^{0}-(i \omega)^{2} \mathbf{M}^{0}=\mathbf{0}
$$


where global coefficient matrices $\mathbf{E}^{0}, \mathbf{E}^{1}, \mathbf{E}^{2}, \mathbf{C}^{0}$ and $\mathbf{M}^{0}$ only depend on the geometry of the near-far-field interface and the reflection coefficient $\alpha_{r}$. They are obtained through assembling all elements' $\mathbf{E}_{e}^{0}, \mathbf{E}_{e}^{1}, \mathbf{E}_{e}^{2}, \mathbf{C}_{e}^{0}$ and $\mathbf{M}_{e}^{0}$ on the near-far-field interface. The matrices $\mathbf{E}_{e}^{0}, \mathbf{E}_{e}^{1}, \mathbf{E}_{e}^{2}, \mathbf{C}_{e}^{0}$ and $\mathbf{M}_{e}^{0}$ corresponding to each element can be evaluated numerically or analytically using the following equations.

$$
\begin{aligned}
\mathbf{E}_{e}^{0} & =\int_{-1}^{1} \int_{-1}^{1} \mathbf{B}^{1 T} \mathbf{B}^{1}|\mathbf{J}| d \eta d \varsigma \\
\mathbf{E}_{e}^{1} & =\int_{-1}^{1} \int_{-1}^{1} \mathbf{B}^{2 T} \mathbf{B}^{1}|\mathbf{J}| d \eta d \varsigma \\
\mathbf{E}_{e}^{2} & =\int_{-1}^{1} \int_{-1}^{1} \mathbf{B}^{2 T} \mathbf{B}^{2}|\mathbf{J}| d \eta d \varsigma \\
\mathbf{M}_{e}^{0} & =\int_{-1}^{1} \int_{-1}^{1} \frac{1}{c^{2}} \mathbf{N}_{f}^{T} \mathbf{N}_{f}|\mathbf{J}| d \eta d \varsigma
\end{aligned}
$$

where the $\mathbf{N}_{f}$ is defined in Eq.(8) and the others $\mathbf{J}, \mathbf{B}^{1}, \mathbf{B}^{2}$ are defined below. The matrix $\mathbf{J}$ is defined as

$$
\mathbf{J}=\left[\begin{array}{ccc}
H & 0 & 0 \\
\frac{d \mathbf{N}_{f}}{d \eta} \mathbf{x} & \frac{d \mathbf{N}_{f}}{d \eta} \mathbf{y} & \frac{d \mathbf{N}_{f}}{d \eta} \mathbf{z} \\
\frac{d \mathbf{N}_{f}}{d \varsigma} \mathbf{x} & \frac{d \mathbf{N}_{f}}{d \varsigma} \mathbf{y} & \frac{d \mathbf{N}_{f}}{d \varsigma} \mathbf{z}
\end{array}\right]
$$

where the symbol $H$ denotes the water depth in the far field and $\mathbf{x}, \mathbf{y}$ and $\mathbf{z}$ are element nodal coordinates column vectors. Due to the fact that $x$ coordinates of all nodes inside the near-far-field interface (vertical surface) are same, the matrix $\mathbf{J}$ becomes

$$
\mathbf{J}=\left[\begin{array}{ccc}
H & 0 & 0 \\
0 & \frac{d \mathbf{N}_{f}}{d \eta} \mathbf{y} & \frac{d \mathbf{N}_{f}}{d \eta} \mathbf{z} \\
0 & \frac{d \mathbf{N}_{f}}{d \varsigma} \mathbf{y} & \frac{d \mathbf{N}_{f}}{d \varsigma} \mathbf{z}
\end{array}\right]
$$

Write the inverse of $\mathbf{J}$ in the following form

$$
\mathbf{J}^{-1}=\left[\begin{array}{lll}
j_{11} & j_{12} & j_{13} \\
j_{21} & j_{22} & j_{23} \\
j_{31} & j_{32} & j_{33}
\end{array}\right]
$$

The components $j_{m n}(m, n=1,2,3)$ can be evaluated by using Eq.(11b). Therefore, the matrix $\mathbf{B}^{1}$ is defined as 


$$
\mathbf{B}^{1}=\left[\begin{array}{l}
j_{11} \\
j_{21} \\
j_{31}
\end{array}\right] \mathbf{N}_{f}
$$

and the matrix $\mathbf{B}^{2}$ is

$$
\mathbf{B}^{2}=\left[\begin{array}{c}
j_{12} \\
j_{22} \\
j_{32}
\end{array}\right] \frac{d \mathbf{N}_{f}}{d \eta}+\left[\begin{array}{l}
j_{13} \\
j_{23} \\
j_{33}
\end{array}\right] \frac{d \mathbf{N}_{f}}{d \varsigma}
$$

Note that Eqs.(10-14) are only the functions of nodal coordinates of elements inside the nearfar-field interface. The matrix $\mathbf{C}_{e}^{0}$ is a zero matrix for elements not adjacent to reservoir bottom inside the near-far-field interface, while for those adjacent to reservoir bottom, $\mathbf{C}_{e}^{0}$ satisfies

$$
\mathbf{C}_{e}^{0}=\frac{1}{c}\left(\frac{1-\alpha_{r}}{1+\alpha_{r}}\right) H \int_{\Gamma_{b}} \mathbf{N}_{f}^{T} \mathbf{N}_{f} d \Gamma_{b}
$$

where the symbol $\Gamma_{b}$ denotes the reservoir bottom of the near-far-field interface, i.e. the line $y=0$ as shown in the Fig.2. Assembling all elements' $\mathbf{E}_{e}^{0}, \mathbf{E}_{e}^{1}, \mathbf{E}_{e}^{2}, \mathbf{C}_{e}^{0}$ and $\mathbf{M}_{e}^{0}$ can yield the global coefficient matrices $\mathbf{E}^{0}, \mathbf{E}^{1}, \mathbf{E}^{2}, \mathbf{C}^{0}$ and $\mathbf{M}^{0}$ in Eq.(9). Details about them can be found in the literatures (Wolf \& Song, 1996b; Li et al., 2008).

For a vertical near-far-field interface as shown in Fig.2, as the matrix $\mathbf{E}^{1}$ was a zero matrix, the dynamic stiffness matrix $\mathbf{S}^{\infty}(\omega)$ in Eq.(9) can be re-written readily as

$$
\mathbf{S}^{\infty}(\omega)=\sqrt{\left(\mathbf{E}^{2}+i \omega \mathbf{C}^{0}-\omega^{2} \mathbf{M}^{0}\right) \mathbf{E}^{0-1}} \mathbf{E}^{0}
$$

where $\omega$ is an excitation frequency. The $\mathbf{S}^{\infty}(\omega)$ can be obtained by the Schur factorization.

\subsection{SBFEM formulation in the time domain}

The corresponding SBFEM formulation of Eq.(7) in the time domain is written as (Wolf \& Song, 1996b)

$$
\mathbf{V}_{n}(t)=\int_{0}^{t} \mathbf{M}^{\infty}(t-\tau) \ddot{\Phi}(\tau) d \tau
$$

in which $\mathbf{M}^{\infty}(t)$ is the dynamic mass matrix of the far field; $\boldsymbol{\Phi}(t)$ and $\mathbf{V}_{n}(t)$ are the corresponding variables of $\boldsymbol{\Phi}(\omega)$ and $\mathbf{V}_{n}(\omega)$ in the time domain, respectively. $\mathbf{M}^{\infty}(t)$ and $\mathbf{S}^{\infty}(\omega) /(i \omega)^{2}$ forms a Fourier transform pair. Upon discretization of Eq.(17) with respect to time and assuming all initial conditions equal to zero, one can get the following equation

$$
\mathbf{V}_{n}^{n}=\mathbf{M}_{1}^{\infty} \dot{\Phi}^{n}+\sum_{j=1}^{n-1}\left(\mathbf{M}_{n-j+1}^{\infty}-\mathbf{M}_{n-j}^{\infty}\right) \dot{\Phi}^{j}
$$

in which $\mathbf{M}_{n-j+1}^{\infty}=\mathbf{M}^{\infty}((n-j+1) \Delta t), \boldsymbol{\Phi}^{j}=\boldsymbol{\Phi}(j \Delta t)$ and $\mathbf{V}_{n}^{n}=\mathbf{V}_{n}(n \Delta t)$ where $\Delta t$ denotes an increment in time step.

Applying the inverse Fourier transformation to Eq. (9) with $\mathbf{E}^{1}=0$ yields 


$$
\int_{0}^{t} \mathbf{m}^{\infty}(t-\tau) \mathbf{m}^{\infty}(\tau) d \tau-\frac{t^{3}}{6} \mathbf{e}^{2}-\frac{t^{2}}{2} \mathbf{c}^{0}-t \mathbf{m}^{0}=0
$$

where $t$ is time and

$$
\begin{aligned}
\mathbf{m}^{\infty}(t) & =\mathbf{U}^{-1 T} \mathbf{M}^{\infty}(t) \mathbf{U}^{-1} \\
\mathbf{e}^{2} & =\mathbf{U}^{-1 T} \mathbf{E}^{2} \mathbf{U}^{-1} \\
\mathbf{m}^{0} & =\mathbf{U}^{-1 T} \mathbf{M}^{0} \mathbf{U}^{-1} \\
\mathbf{c}^{0} & =\mathbf{U}^{-1 T} \mathbf{C}^{0} \mathbf{U}^{-1}
\end{aligned}
$$

in which $\mathbf{U}$ satisfies

$$
\mathbf{E}^{0}=\mathbf{U}^{T} \mathbf{U}
$$

A procedure (Wolf \& Song, 1996b) was presented to evaluate the dynamic mass matrix $\mathbf{M}^{\infty}(t)$ at different time $t$ governed by the convolution integral Eq.(19). In that procedure, discretization of Eq.(19) with respect to time was implemented, and an algebraic Riccati equation for evaluating $\mathbf{M}^{\infty}(t=\Delta t)$ at first time step and a Lyapunov equation for evaluating $\mathbf{M}^{\infty}(t=j \Delta t)$ at other $\mathrm{jth}$ time steps were formed, respectively. The $\mathbf{M}^{\infty}(t=j \Delta t)$ at any time was obtained by utilizing Schur factorization to solve these two types of equations. When the coefficient matrix $c^{0}=0$, a simple diagonal procedure (Li, 2009) can be adopted to evaluate the $\mathbf{M}^{\infty}(t)$, which can avoid Schur factorization and solving Riccati equation and Lyapunov equation.

\section{FEM-SBFEM coupling formulation of reservoir}

To obtain the response of dam-reservoir system, the near-field fluid domain is discretized into an assemblage of finite elements. The corresponding finite-element governing equation of Eq.(1) for the near-field domain can be expressed as

$$
\left[\begin{array}{lll}
\mathbf{m}_{11} & \mathbf{m}_{12} & \mathbf{m}_{13} \\
\mathbf{m}_{21} & \mathbf{m}_{22} & \mathbf{m}_{23} \\
\mathbf{m}_{31} & \mathbf{m}_{32} & \mathbf{m}_{33}
\end{array}\right]\left\{\begin{array}{l}
\ddot{\boldsymbol{\Phi}}_{1} \\
\ddot{\boldsymbol{\Phi}}_{2} \\
\ddot{\boldsymbol{\Phi}}_{3}
\end{array}\right\}+\left[\begin{array}{lll}
\mathbf{k}_{11} & \mathbf{k}_{12} & \mathbf{k}_{13} \\
\mathbf{k}_{21} & \mathbf{k}_{22} & \mathbf{k}_{23} \\
\mathbf{k}_{31} & \mathbf{k}_{32} & \mathbf{k}_{33}
\end{array}\right]\left\{\begin{array}{l}
\boldsymbol{\Phi}_{1} \\
\boldsymbol{\Phi}_{2} \\
\boldsymbol{\Phi}_{3}
\end{array}\right\}=\left\{\begin{array}{c}
\mathbf{V}_{n 1} \\
\mathbf{V}_{12}^{\prime} \\
\mathbf{V}_{n 3}
\end{array}\right\}
$$

where the global mass matrix $\mathbf{m}$, the global stiffness matrix $\mathbf{k}$ and the global vector $\mathbf{V}_{n}$ are treated in the standard manner as in the traditional FE procedures; the subscripts 1 and 2 refer to nodal variables at the dam-reservoir interface and the near-far-field interface, respectively, while the subscript 3 refers to other interior nodal variables in the near-field fluid. At the near-far-field interface, the near-field FEM-domain couples with the far-field SBFEM-domain. The kinematic continuity condition requires that both fields have the same normal velocity at the near-far-field interface. Hence, one has

$$
-\mathbf{V}_{n 2}^{\prime}=\mathbf{V}_{n}
$$


In the frequency domain, using Eqs.(7, 16, 25, 26) yields

$$
\begin{aligned}
& {\left[\begin{array}{lll}
\mathbf{m}_{11} & \mathbf{m}_{12} & \mathbf{m}_{13} \\
\mathbf{m}_{21} & \mathbf{m}_{22} & \mathbf{m}_{23} \\
\mathbf{m}_{31} & \mathbf{m}_{32} & \mathbf{m}_{33}
\end{array}\right]\left\{\begin{array}{l}
\ddot{\boldsymbol{\Phi}}_{1} \\
\left.\ddot{\boldsymbol{\Phi}}_{2}\right\} \\
\ddot{\boldsymbol{\Phi}}_{3}
\end{array}\right\}+} \\
& {\left[\begin{array}{lll}
\mathbf{k}_{11} & \mathbf{k}_{12} & \mathbf{k}_{13} \\
\mathbf{k}_{21} & \mathbf{k}_{22}+\sqrt{\left(\mathbf{E}^{2}+i \omega \mathbf{C}^{0}-\omega^{2} \mathbf{M}^{0}\right) \mathbf{E}^{0-1}} \mathbf{E}^{0} & \mathbf{k}_{23} \\
\mathbf{k}_{31} & \mathbf{k}_{32} & \mathbf{k}_{33}
\end{array}\right]\left\{\begin{array}{l}
\boldsymbol{\Phi}_{1} \\
\boldsymbol{\Phi}_{2} \\
\boldsymbol{\Phi}_{3}
\end{array}\right\}=\left\{\begin{array}{c}
\mathbf{V}_{n 1} \\
\mathbf{0} \\
\mathbf{V}_{n 3}
\end{array}\right\}}
\end{aligned}
$$

For a harmonic response with an exciting frequency $\omega$,

$$
\mathbf{\Phi}=\overline{\mathbf{\Phi}} e^{i \omega t}
$$

Substituting Eq.(28) into Eq.(27) leads to the FEM-SBFEM coupling equation of a reservoir to solve the harmonic response of a reservoir, i.e.

$$
\begin{gathered}
-\omega^{2}\left[\begin{array}{lll}
\mathbf{m}_{11} & \mathbf{m}_{12} & \mathbf{m}_{13} \\
\mathbf{m}_{21} & \mathbf{m}_{22} & \mathbf{m}_{23} \\
\mathbf{m}_{31} & \mathbf{m}_{32} & \mathbf{m}_{33}
\end{array}\right]+ \\
\left.\left[\begin{array}{lll}
\mathbf{k}_{11} & \mathbf{k}_{12} & \mathbf{k}_{13} \\
\mathbf{k}_{21} & \mathbf{k}_{22}+\sqrt{\left(\mathbf{E}^{2}+i \omega \mathbf{C}^{0}-\omega^{2} \mathbf{M}^{0}\right) \mathbf{E}^{0-1}} \mathbf{E}^{0} & \mathbf{k}_{23} \\
\mathbf{k}_{31} & \mathbf{k}_{32} & \mathbf{k}_{33}
\end{array}\right]\right)\left\{\begin{array}{l}
\overline{\mathbf{\Phi}}_{1} \\
\overline{\mathbf{\Phi}}_{2} \\
\overline{\boldsymbol{\Phi}}_{3}
\end{array}\right\} e^{i \omega t}=\left\{\begin{array}{c}
\mathbf{V}_{n 1} \\
\mathbf{0} \\
\mathbf{V}_{n 3}
\end{array}\right\}
\end{gathered}
$$

Eq.(29) can be solved for any frequency $\omega$.

In the time domain, using Eqs.(17, 18, 25, 26) yields the FEM-SBFEM coupling equation of a reservoir to solve the transient response of a reservoir, i.e.

$$
\begin{gathered}
{\left[\begin{array}{lll}
\mathbf{m}_{11} & \mathbf{m}_{12} & \mathbf{m}_{13} \\
\mathbf{m}_{21} & \mathbf{m}_{22} & \mathbf{m}_{23} \\
\mathbf{m}_{31} & \mathbf{m}_{32} & \mathbf{m}_{33}
\end{array}\right]\left\{\begin{array}{l}
\ddot{\boldsymbol{\Phi}}_{1}^{n} \\
\ddot{\boldsymbol{\Phi}}_{2}^{n} \\
\ddot{\boldsymbol{\Phi}}_{3}^{n}
\end{array}\right\}+\left[\begin{array}{ccc}
\mathbf{0} & \mathbf{0} & \mathbf{0} \\
\mathbf{0} & \mathbf{M}_{1}^{\infty} & \mathbf{0} \\
\mathbf{0} & \mathbf{0} & \mathbf{0}
\end{array}\right]\left\{\begin{array}{l}
\dot{\boldsymbol{\Phi}}_{1}^{n} \\
\dot{\boldsymbol{\Phi}}_{2}^{n} \\
\dot{\boldsymbol{\Phi}}_{3}^{n}
\end{array}\right\}} \\
\mathbf{V}_{n 1}^{n} \\
+\left[\begin{array}{lll}
\mathbf{k}_{11} & \mathbf{k}_{12} & \mathbf{k}_{13} \\
\mathbf{k}_{21} & \mathbf{k}_{22} & \mathbf{k}_{23} \\
\mathbf{k}_{31} & \mathbf{k}_{32} & \mathbf{k}_{33}
\end{array}\right]\left\{\begin{array}{l}
\boldsymbol{\Phi}_{1}^{n} \\
\boldsymbol{\Phi}_{2}^{n} \\
\boldsymbol{\Phi}_{3}^{n}
\end{array}\right\}=\left\{\begin{array}{c}
n-1 \\
-\sum_{j=1}^{n}\left(\mathbf{M}_{n-j+1}^{\infty}-\mathbf{M}_{n-j}^{\infty}\right) \dot{\boldsymbol{\Phi}}_{2}^{j} \\
\mathbf{V}_{n 3}^{n}
\end{array}\right\}
\end{gathered}
$$

where the superscript $n$ denotes the instant at time $t=n \Delta t$. Note that a damping matrix appears on the left hand side of Eq.(30). It can be regarded as the damping effect derived from the far-field medium and imposed on the dam-reservoir system. As the near-field domain is modeled by FEM, Eqs. $(29,30)$ are suitable for a reservoir with any arbitrary geometry shape. 


\section{Numerical examples}

\subsection{Harmonic response of reservoir}

Two-dimensional dam-reservoir systems subjected to horizontal harmonic ground accelerations $a=\bar{a} e^{i \omega t}$ in the upstream direction were studied. For simplicity, here the dam was assumed to be rigid.

\subsubsection{Vertical dam}

For a rigid dam-reservoir system with a vertical upstream face as shown in Fig.3, the whole reservoir was flat so that the whole reservoir was modeled by the far field alone. This example's aim was only to test the correctness and efficiency of the SBFEM in Eqs. $(7,8,16)$ of the far field. The whole reservoir was discretized by the SBFEM alone using 10 and 20 3noded SBFEM elements, respectively. The hydrodynamic pressure acting on the damreservoir interface from a reflection coefficient $\alpha_{r}=0.95$ and these two mesh densities was plotted in Fig.4. The coefficient $C_{p}$ was defined as $|p| /(\rho \bar{a} H)$ and $\omega_{1}=c \pi /(2 H)$, where $p$ denoted the amplitude of hydrodynamic pressure acting on the dam-reservoir interface.

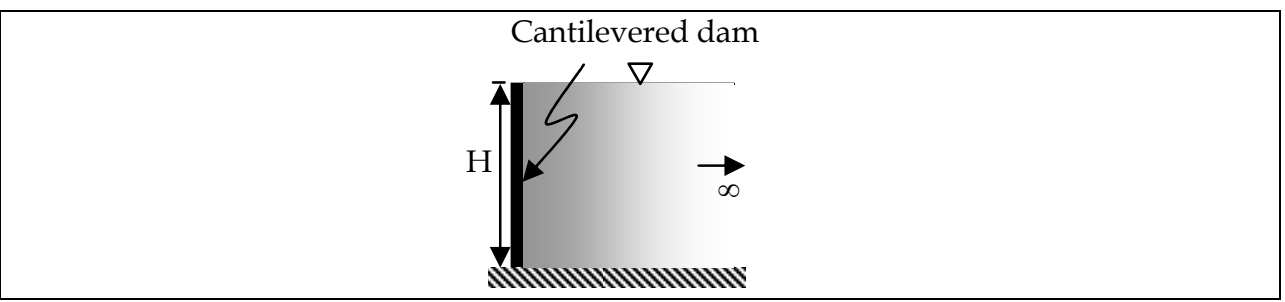

Fig. 3. Vertical dam-reservoir system

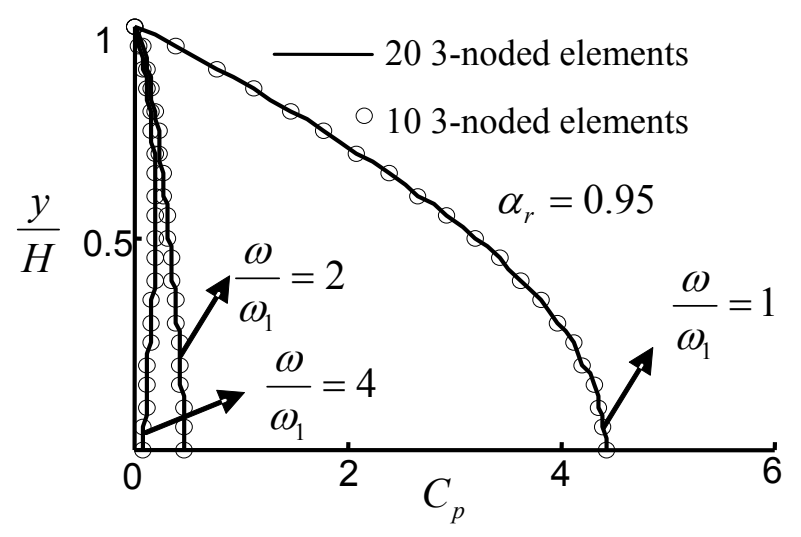

Fig. 4. Hydrodynamic pressure on vertical dam-reservoir interface from different meshes 
Results from different mesh densities were the same. The hydrodynamic pressure obtained by using 10 3-noded SBFEM elements and the corresponding analytical solutions (Weber, 1994) corresponding to different $\alpha_{r}$ were plotted in Fig.5. The SBFEM solutions were the exact same to the analytical solutions. Furthermore, a $\omega-C_{p}$ figure of a point located at $y=0.6 \mathrm{H}$ corresponding to $\alpha_{r}=0.8$ was shown in Fig.6. The SBFEM solution and the analytical solution (Weber, 1994) were the same.
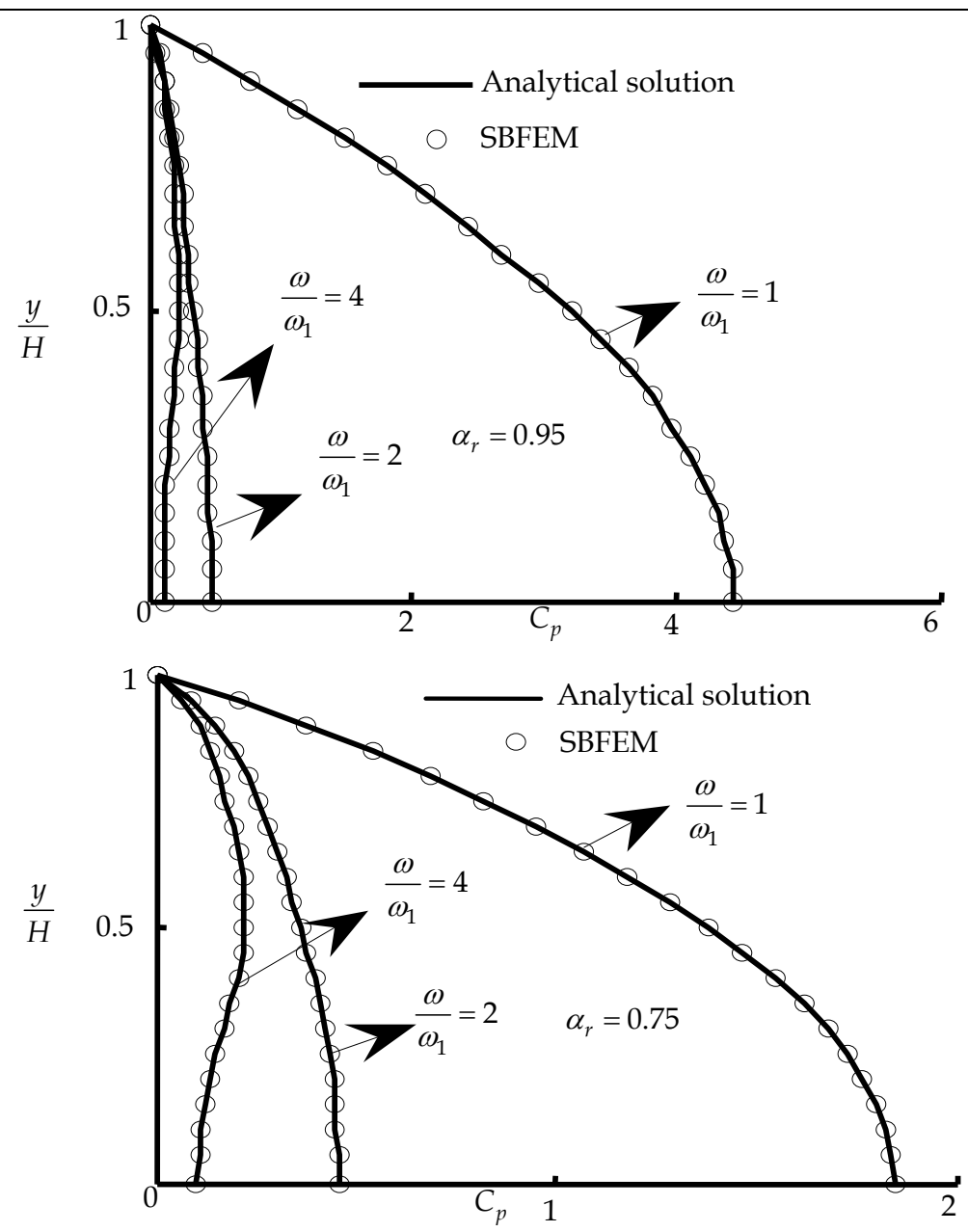

Fig. 5. Hydrodynamic pressures on vertical dam-reservoir interface caused by different $\alpha_{r}$ 


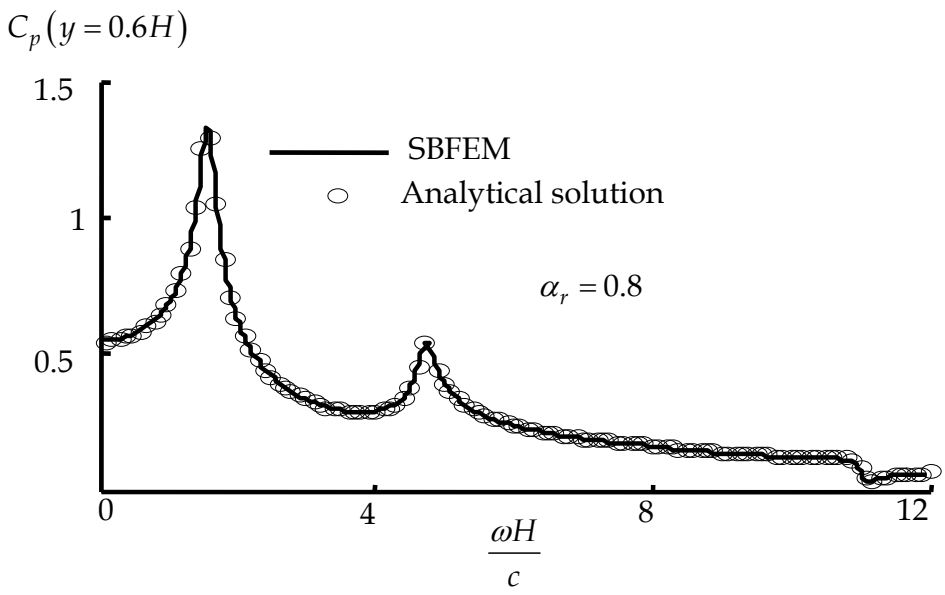

Fig. 6. $C_{p}(y=0.6 H)$ for different $\omega$

\subsubsection{Gravity dam}

A gravity dam shown in Fig.7 was considered to verify the correctness and efficiency of the FEM-SBFEM coupling formulation in Eq.(29). The near field was chosen as the domain with a very small distance $L=0.001 \mathrm{H}$ away from the heel of dam and was discretized by 8-noded

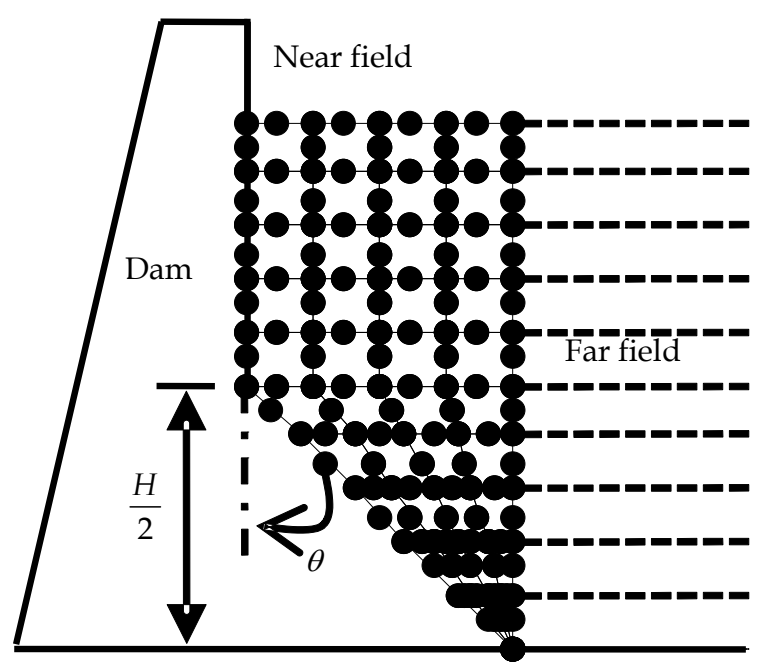

Fig. 7. Meshes of gravity dam with multi-sloping faces and $\theta=45^{0}$ 
isoparametric acoustic fluid finite elements, while the far field was still modeled by 10 3noded SBFEM elements. Their meshes were shown in Fig.7. Solutions from Eq.(29) and the literature (Sharan, 1992) were plotted in Fig.8. Results obtained by Eq.(29) were in excellent agreement with Sharan's results.

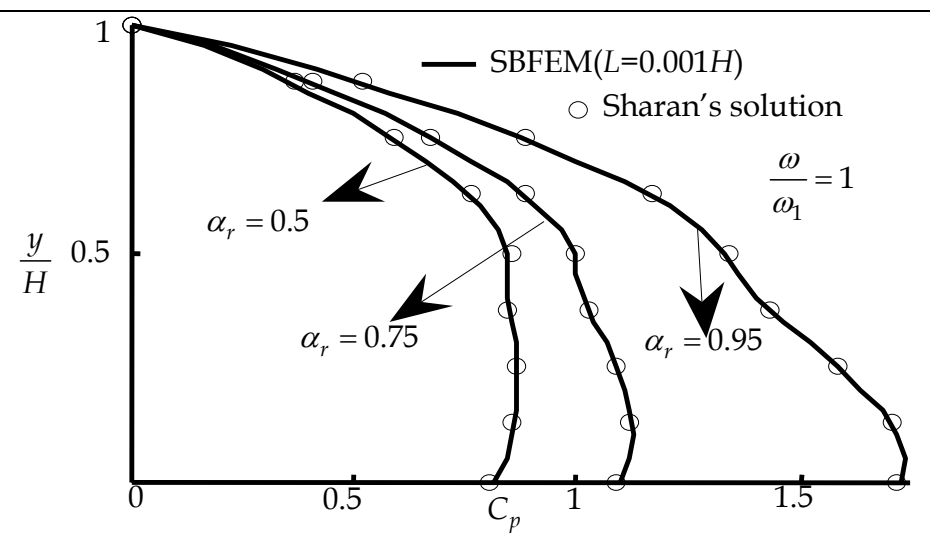

Fig. 8. Hydrodynamic pressure acting on gravity dam

\subsection{Transient response of dam-reservoir system}

Consider transient responses of dam-reservoir systems where dams were subjected to horizontal ground acceleration excitations shown in Fig.9. In the transient analysis, only the linear behavior was considered, the free surface wave effects and the reservoir bottom absorption were ignored, and the damping of dams was excluded. Dams were discretized by the FEM, while the response of the reservoir was solved by Eq.(30). The FE equation of dam and Eq.(30) was solved by Newmark's time-integration scheme with Newmark integration parameters $\alpha=0.25$ and $\delta=0.5$. An iteration scheme (Fan et al., 2005) was adopted to obtain the response of the dam-reservoir interaction problems.
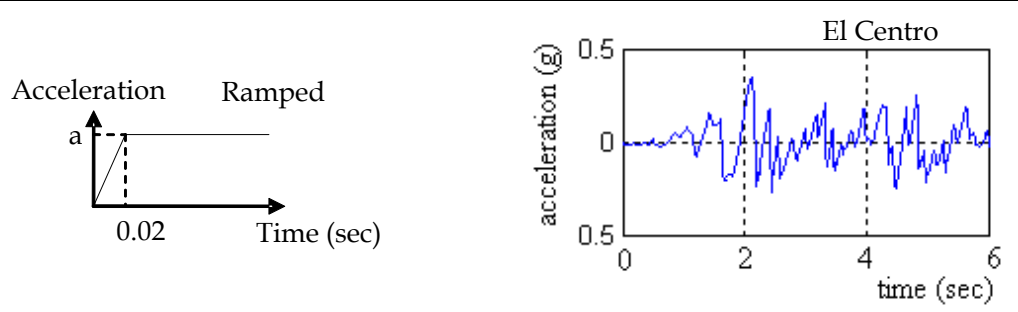

Fig. 9. Horizontal acceleration excitations 


\subsubsection{Vertical dam}

As the cross section of the vertical dam-system as shown in Fig.3 was uniform, a near-field fluid domain was not necessary and the whole reservoir was modeled by a far-field domain alone. Sound speed in the reservoir is $1438.656 \mathrm{~m} / \mathrm{s}$ and the fluid density $\rho$ is $1000 \mathrm{~kg} / \mathrm{m}^{3}$. The weight per unit length of the cantilevered dam was $36000 \mathrm{~kg} / \mathrm{m}$. The height of the cantilevered dam $H$ was $180 \mathrm{~m}$. The dam was modeled by 20 numbers of simple 2-noded beam elements with rigidity $E I\left(=9.646826 \times 10^{13} \mathrm{Nm}^{2}\right)$, while the whole fluid domain was modeled by 10 numbers of 3-noded SBFEM elements, whose nodes matched side by side with nodes of the dam. In this problem, the shear deformation effects were not included in the 2-noded beam elements. Time step increment was $0.005 \mathrm{sec}$. The pressure at the heel of dam subjected to the ramped horizontal acceleration shown in Fig.9 was plotted in Fig.10 and Fig.11. Analytical solutions of deformable and rigid dams were from the literature (Tsai et al., 1990) and the literature (Weber, 1994), respectively. In Fig.11, analytical solutions (Weber, 1994), solutions from the SBFEM in the full matrix form (Wolf \& Song, 1996b) and solutions from the SBFEM in the diagonal matrix form (Li, 2009) were plotted with circles, rectangles and solid line, respectively. Solutions from the SBFEM and analytical solutions were the same. In the literature $(\mathrm{Li}, 2009)$, it was found that diagonal SBFEM formulations need much less computational costs than those in the full matrix.

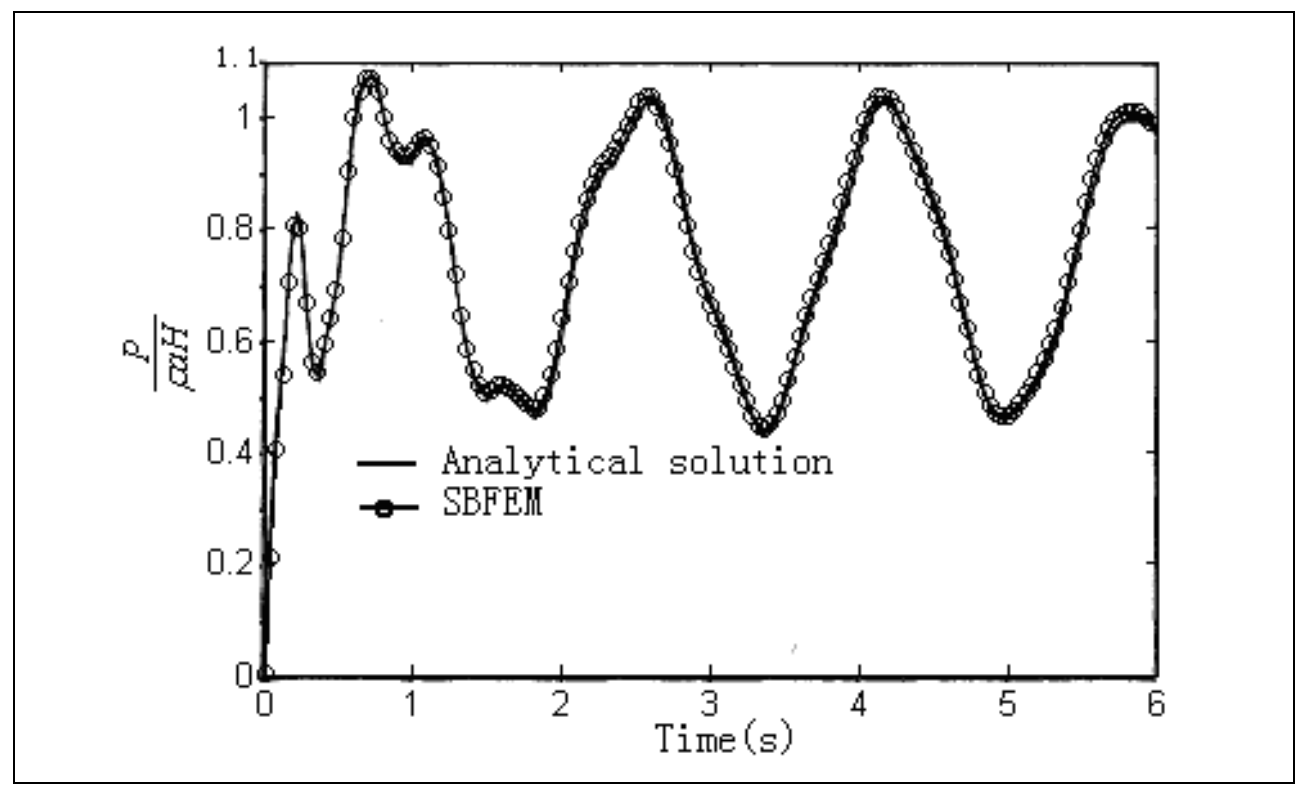

Fig. 10. Pressure at the heel of deformable dam subjected to ramped horizontal acceleration 


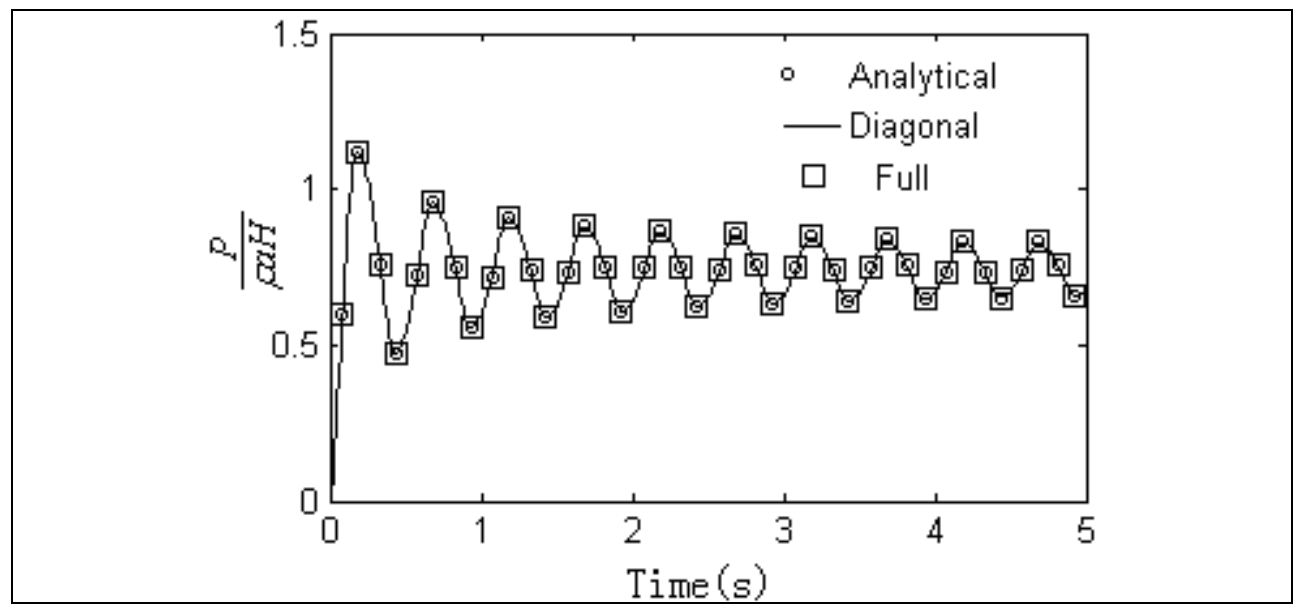

Fig. 11. Pressure at the heel of rigid dam subjected to ramped horizontal acceleration

\subsubsection{Gravity dam}

This example was analyzed to verify the accuracy and efficiency of the FEM-SBFEM coupling formulation for a dam-reservoir system having arbitrary slopes at the damreservoir interface. The density, Poisson's ratio and Young's modulus of the deformable dam are $2400 \mathrm{~kg} / \mathrm{m}^{3}, 0.2$ and $2.5 \times 10^{10} \mathrm{~N} / \mathrm{m}^{2}$, respectively. The fluid density $\rho$ is $1000 \mathrm{~kg} / \mathrm{m}^{3}$ and wave speed in the fluid is $1438.656 \mathrm{~m} / \mathrm{s}$. The height of the dam $H$ is $120 \mathrm{~m}$. A typical gravitydam-reservoir system and its FEM and SBFEM meshes were shown in Fig.12. The dam and the near-field fluid were discretized by FEM, while the far-field fluid was discretized by the SBFEM. 40 numbers and 20 numbers of 8-noded elements were used to model the dam and the near-field fluid domain, respectively, while 10 numbers of 3-noded SBFEM elements were employed to model the whole far-field fluid domain. Note that the size of the nearfield fluid domain can be very small compared to those used in other methods. In this example, the distance between the heel of the dam and the near-far-field interface was $6 \mathrm{~m}$

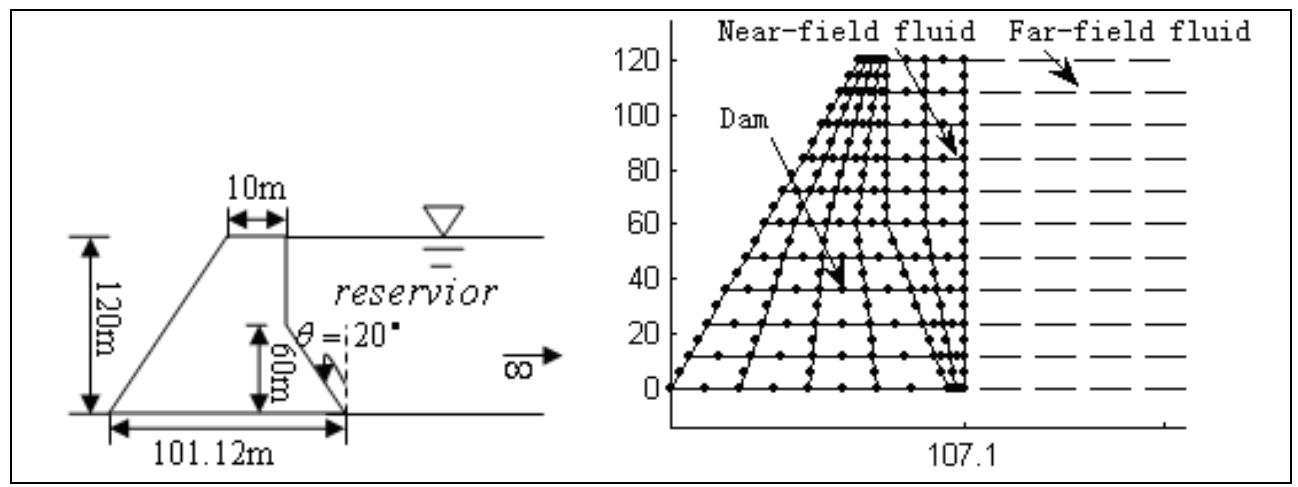

Fig. 12. Gravity dam-reservoir system and its FEM-SBFEM mesh 
$(=0.05 H)$. The pressure at the heel of the gravity dam caused by the horizontal ground acceleration shown in Fig.9 was plotted in Fig.13. The time increment was 0.002sec. Results from SBFEM were very close to solutions from the sub-structures method (Tsai \& Li, 1991). The displacements at the top of vertical and gravity dams subjected to a ramped horizontal acceleration were plotted in Fig.14. The displacement solutions of vertical dam from the SBFEM were the same with analytical solutions (Tsai et al., 1990). Fig.15 showed the displacement at the top of gravity dam subjected to the El Centro horizontal acceleration. At early time, the displacements obtained by the present method agreed well with substructure method's results (Tsai et al., 1990), especially at early time.

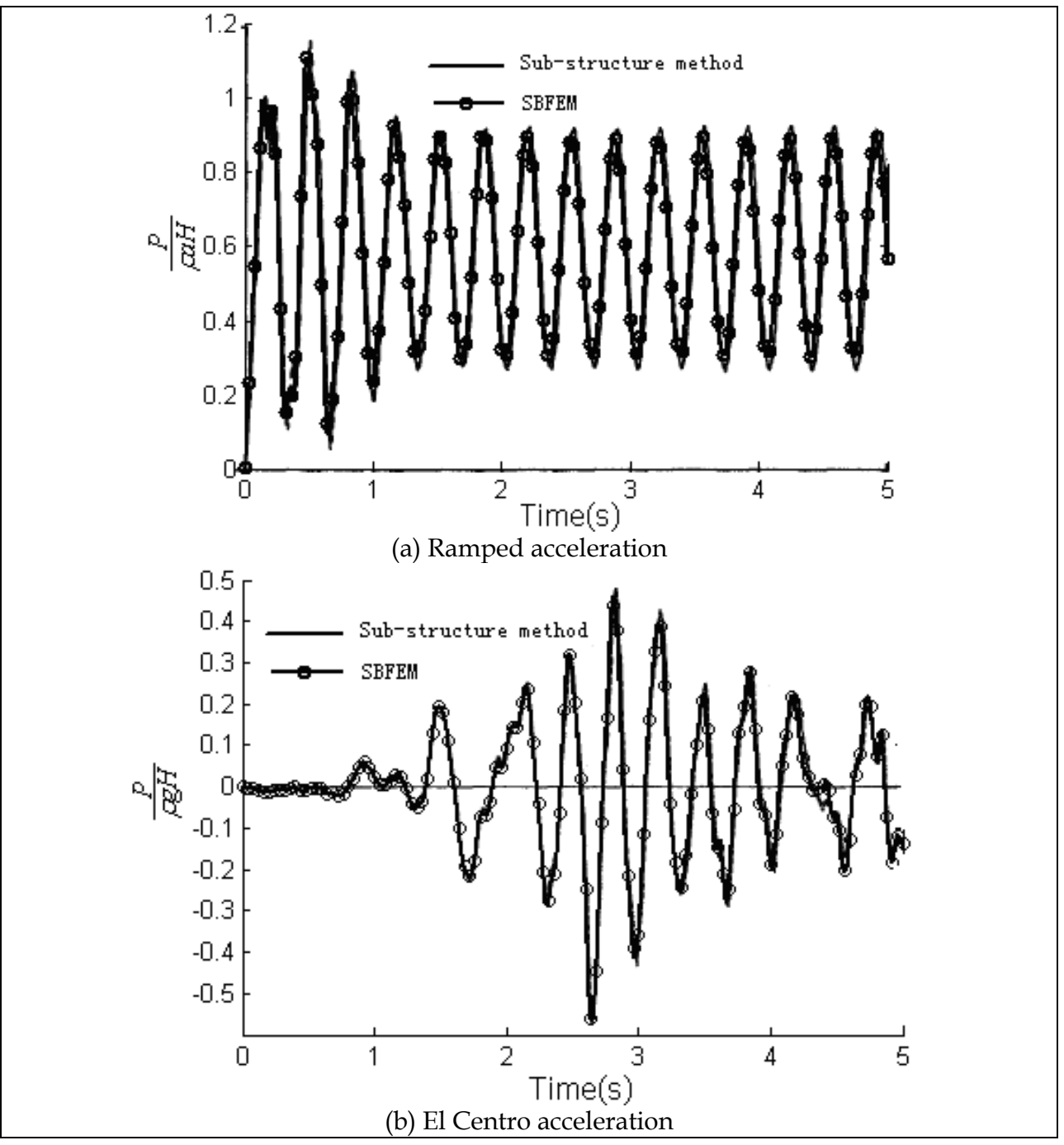

Fig. 13. Pressure at the heel of gravity dam subjected to horizontal acceleration 


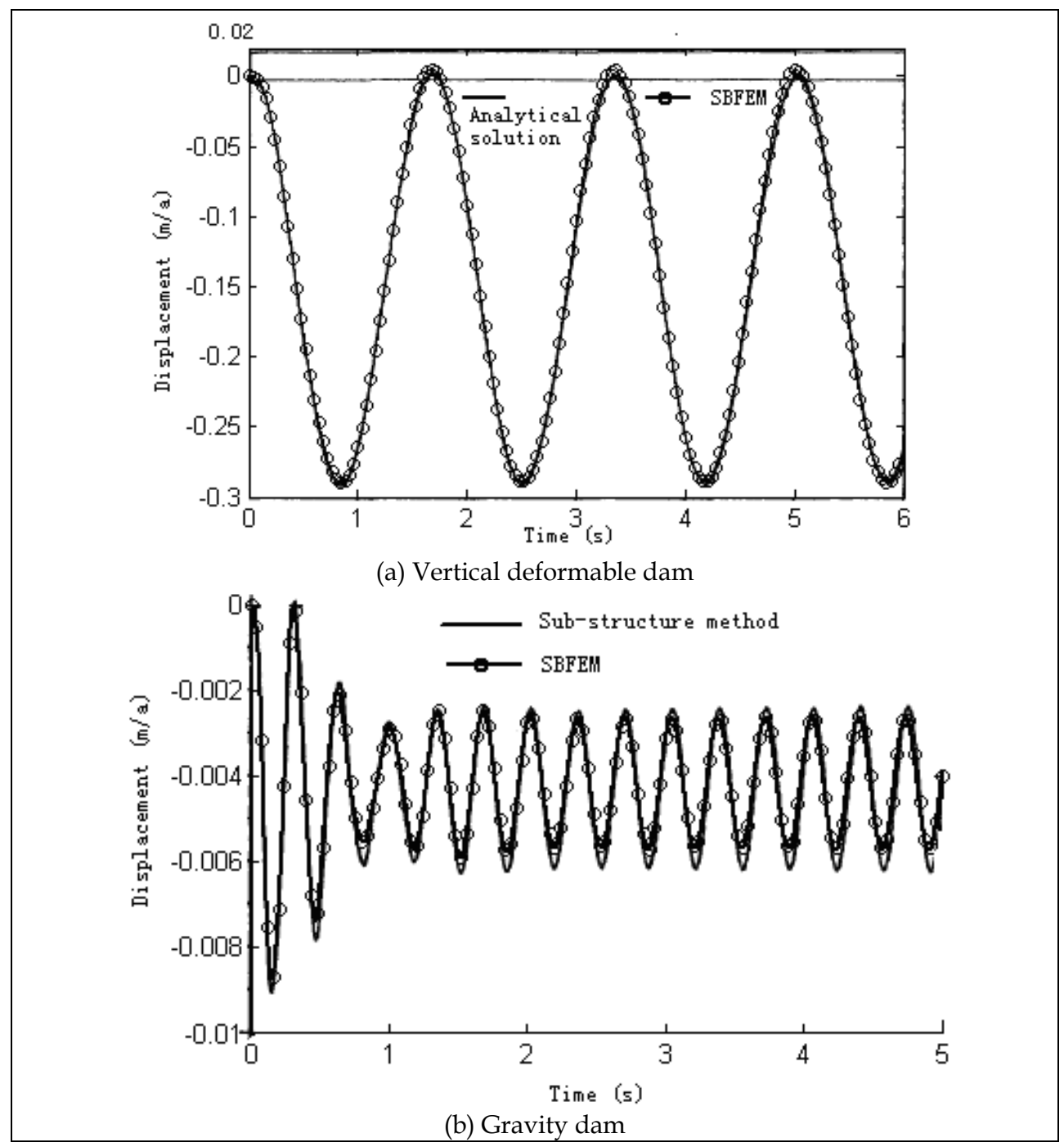

Fig. 14. Displacement at top of dam subjected to ramped horizontal acceleration 


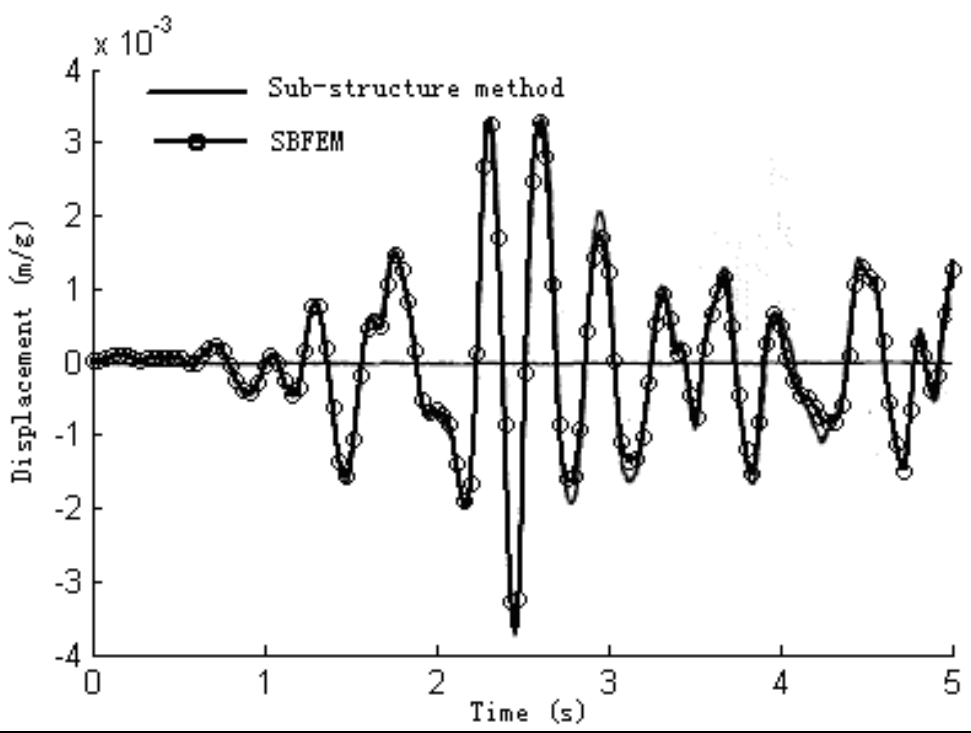

Fig. 15. Displacement at top of gravity dam subjected to El Centro horizontal acceleration

\section{Conclusion}

Aiming for dam-reservoir system problems subjected to horizontal ground motions, this chapter presented the SBFEM formulations in the frequency and time domain and its corresponding FEM-SBFEM coupling formulations to evaluate the hydrodynamic pressure of the reservoir through dividing the reservoir into a near field and far field, where the dam and the near field were modeled by FEM and the far field was discretized by the SBFEM. The SBFEM uses the dynamic stiffness matrix and the dynamic mass matrix to describe the dynamic characteristics of the far field in the frequency and time domain, respectively. The merits of the SBFEM in representing the semi-infinite reservoir were illustrated through comparisons against benchmark solutions. Numerical results showed that its accuracy and efficiency of the FEM-SBFEM formulation to obtain the harmonic and transient analysis of a dam-reservoir system. Of note, the SBFEM is a semi-analytical method. Its solution in the radial direction is analytical so that only a near field with a small volume is required. Compared to the sub-structure method, its formulations are in a simpler mathematical form and can be coupled with FEM easily and seamlessly.

\section{Acknowledgments}

This research is supported by the National Natural Science Foundation of China (No. 10902060) and China Postdoctoral Science Foundation (201003123), for which the author is grateful. 


\section{References}

Bazyar, M.H. \& Song, C.M. (2008). A continued-fraction-based high-order transmitting boundary for wave propagation in unbounded domains of arbitrary geometry. International Journal for Numerical Methods in Engineering, Vol.74, pp.209-237

Czygan, O. \& Von, Estorff, O. (2002). Fluid-structure interaction by coupling BEM and nonlinear FEM. Engineering Analysis with Boundary Elements, Vol.26, pp.773-779

Dominguez, J ; Gallego, R. \& Japon, B.R. (1997). Effects of porous sediments on seismic response of concrete gravity dams. Journal of Engineering Mechanics - ASCE, Vol.123, pp.302-311

Dominguez, J. \& Maeso, O. (1993). Earthquake analysis of arch dams II. Dam-waterfoundation interaction. Journal of Engineering Mechanics - ASCE, Vol.119, pp.513-530

Ekevid, T. \& Wiberg, N.E. (2002). Wave propagation related to high-speed train - A scaled boundary FE-approach for unbounded domains. Computer Methods in Applied Mechanics and Engineering, Vol.191, pp.3947-3964

Fan, S.C. ; Li, S.M. \& Yu, G.Y. (2005). Dynamic fluid-structure interaction analysis using boundary finite element method-finite element method. Journal of Applied Mechanics - Transactions of the ASME, Vol.72, pp.591-598

Fan, S.C. \& Li, S.M. (2008). Boundary finite-element method coupling finite-element method for steady-state analyses of dam-reservoir systems. Journal of Engineering Mechanics - ASCE, Vol.134, pp.133-142

Gogoi, I. \& Maity, D. (2006). A non-reflecting boundary condition for the finite element modeling of infinite reservoir with layered sediment. Advances in Water Resources, Vol.29, pp.1515-1527

Kucukarslan, S .; Coskun, S.B. \& Taskin, B. (2005). Transient analysis of dam-reservoir interaction including the reservoir bottom effects. Journal of Fluids and Structures, Vol.20, pp.1073-1084

Li, S.M. \& Fan, S.C. (2007). Parametric analysis of a submerged cylindrical shell subjected to shock waves. China Ocean Engineering, Vol.21, pp.125-136

Li, S.M. ; Liang, H. \& Li, A.M. (2008). A semi-analytical solution for characteristics of a damreservoir system with absorptive reservoir bottom. Journal of Hydrodynamics, Vol.20, pp.727-734.

Li, S.M. (2009). Diagonalization procedure for scaled boundary finite element method in modelling semi-infinite reservoir with uniform cross section. International Journal for Numerical Methods in Engineering, Vol.80, pp.596-608

Maity, D. \& Bhattacharyya, S.K. (1999). Time-domain analysis of infinite reservoir by finite element method using a novel far-boundary condition. Finite Elements in Analysis and Design, Vol.32, pp.85-96

Paronesso, A. \& Wolf, J.P. (1995). Global lumped-parameter model with physical representation for unbounded medium. Earthquake Engineering and Structural Dynamics, Vol.24, pp.637-654

Paronesso, A. \& Wolf, J.P. (1998). Recursive evaluation of interaction forces and property matrices from unit-impulse response functions of unbounded medium based on balancing approximation. Earthquake Engineering and Structural Dynamics, Vol.27, pp.609-618

Sharan, S.K. (1992). Efficient finite element analysis of hydrodynamic pressure on dams. Computers and Structures, Vol.42, No.5, pp.713-723 
Sharan, S.K. (1987). Time-domain analysis of infinite fluid vibration. International Journal for Numerical Methods in Engineering, Vol.24, pp.945-958

Song, C.M. \& Bazyar, M.H. (2007). A boundary condition in Pade series for frequencydomain solution of wave propagation in unbounded domains. International Journal for Numerical Methods in Engineering, Vol.69, pp.2330-2358

Song, C.M. \& Wolf, J.P. (1996). Consistent infinitesimal finite-element cell method: Threedimensional vector wave equation. International Journal for Numerical Methods in Engineering, Vol.39, pp.2189-2208

Song, C.M. \& Wolf, J.P. (2002). Semi-analytical representation of stress singularities as occurring in cracks in anisotropic multi-materials with the scaled boundary finiteelement method. Computers and Structures, Vol.80, pp.183-197

Song, C.M. (2004). A super-element for crack analysis in the time domain. International Journal for Numerical Methods in Engineering, Vol.61, pp.1332-1357

Tsai, C.S. ; Lee, G.C. \& Ketter, R.L. (1990). A semi-analytical method for time-domain analyses of dam-reservoir interactions. International Journal for Numerical Methods in Engineering, Vol.29, pp.913-933

Tsai, C.S. \& Lee, G.C. (1987). Arch dam fluid interactions - by FEM-BEM and sub-structure concept. International Journal for Numerical Methods in Engineering, Vol.24, pp.23672388

Tsai, C.S. \& Lee, G.C. (1991). Time-domain analyses of dam-reservoir system II. Substructure method. Journal of Engineering Mechanics - ASCE, Vol.117, pp.2007-2026

Weber, B. (1994). Rational transmitting boundaries for time-domain analysis of dam-reservoir interaction, Birkhauser Verlag, ISBN-10, 0817651233, Basel, Boston

Wolf, J.P. \& Song, C.M. (1996a). Consistent infinitesimal finite element cell method: Three dimensional scalar wave equation. Journal of Applied Mechanics - Transactions of the ASME, Vol.63, pp.650-654

Wolf, J.P. \& Song, C.M. (1996b). Finite-Element Modeling of Unbounded Media, Wiley, ISBN 978-0-471-96134-5, Chichester

Yan, J.Y.; Zhang, C.H. \& Jin, F. (2004). A coupling procedure of FE and SBFE for soilstructure interaction in the time domain. International Journal for Numerical Methods in Engineering, Vol.59, pp.1453-1471

Yang, Z.J. \& Deeks, A.J. (2007). Fully-automatic modelling of cohesive crack growth using a finite element-scaled boundary finite element coupled method. Engineering Fracture Mechanics, Vol.74, pp.2547-2573 


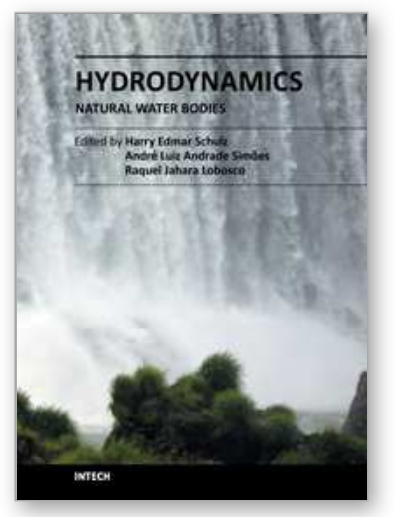

\author{
Hydrodynamics - Natural Water Bodies \\ Edited by Prof. Harry Schulz
}

ISBN 978-953-307-893-9

Hard cover, 286 pages

Publisher InTech

Published online 05, January, 2012

Published in print edition January, 2012

The knowledge of the characteristics of the fluids and their ability to transport substances and physical properties is relevant for us. However, the quantification of the movements of fluids is a complex task, and when considering natural flows, occurring in large scales (rivers, lakes, oceans), this complexity is evidenced. This book presents conclusions about different aspects of flows in natural water bodies, such as the evolution of plumes, the transport of sediments, air-water mixtures, among others. It contains thirteen chapters, organized in four sections: Tidal and Wave Dynamics: Rivers, Lakes and Reservoirs, Tidal and Wave Dynamics: Seas and Oceans, Tidal and Wave Dynamics: Estuaries and Bays, and Multiphase Phenomena: Air-Water Flows and Sediments. The chapters present conceptual arguments, experimental and numerical results, showing practical applications of the methods and tools of Hydrodynamics.

\title{
How to reference
}

In order to correctly reference this scholarly work, feel free to copy and paste the following:

Shangming Li (2012). Hydrodynamic Pressure Evaluation of Reservoir Subjected to Ground Excitation Based on SBFEM, Hydrodynamics - Natural Water Bodies, Prof. Harry Schulz (Ed.), ISBN: 978-953-307-893-9, InTech, Available from: http://www.intechopen.com/books/hydrodynamics-natural-water-bodies/hydrodynamicpressure-evaluation-of-reservoir-subjected-to-ground-excitation-based-on-sbfem

\section{INTECH}

open science | open minds

\author{
InTech Europe \\ University Campus STeP Ri \\ Slavka Krautzeka 83/A \\ 51000 Rijeka, Croatia \\ Phone: +385 (51) 770447 \\ Fax: +385 (51) 686166 \\ www.intechopen.com
}

\author{
InTech China \\ Unit 405, Office Block, Hotel Equatorial Shanghai \\ No.65, Yan An Road (West), Shanghai, 200040, China \\ 中国上海市延安西路65号上海国际贵都大饭店办公楼 405 单元 \\ Phone: +86-21-62489820 \\ Fax: +86-21-62489821
}


(C) 2012 The Author(s). Licensee IntechOpen. This is an open access article distributed under the terms of the Creative Commons Attribution 3.0 License, which permits unrestricted use, distribution, and reproduction in any medium, provided the original work is properly cited. 